\title{
The enigmatic WR46: A binary or a pulsator in disguise
}

\section{Interpretation}

\author{
P. M. Veen ${ }^{1}$, A. M. van Genderen ${ }^{1}$, and K. A. van der Hucht ${ }^{2}$ \\ 1 Leiden Observatory, Postbus 9513, 2300 RA Leiden, The Netherlands \\ 2 Space Research Organization Netherlands, Sorbonnelaan 2, 3584 CA Utrecht, The Netherlands
}

Received 1 September 2000 / Accepted 5 November 2001

\begin{abstract}
Photometric and spectroscopic monitoring campaigns of WR 46 (WN3p), as presented in Veen et al. (2002a,b; hereafter Papers I and II, respectively), yield the following results. The light- and colour variations reveal a dominant single-wave period of $P_{\mathrm{sw}}^{89}=0.1412 \mathrm{~d}$ in 1989 , and $P_{\mathrm{sw}}^{91}=0.1363 \mathrm{~d}$ in 1991 . Because of a small difference in the minima, this periodicity may be a double-wave phenomenon $\left(P_{\mathrm{dw}}\right)$. The line fluxes vary in concert with the magnitudes. The significant difference of the periods can be either due to the occurence of two distinct periods, or due to a gradual change of the periodicity. A gradual brightening of the system of 0 . 12 appeared to accompany the period change. In addition, the light variations in 1989 show strong evidence for an additional period $P_{\mathrm{x}}=0.2304 \mathrm{~d}$. Generally, the radial velocities show a cyclic variability on a time scale of the photometric double-wave. However, often they do not vary at all. The observed variability confirms the Population I WR nature of the light source, as noted independently by Marchenko et al. (2000). In the present paper, we first show how the photometric double-wave variability can be interpreted as a rotating ellipsoidal density distribution in the stellar wind. Subsequently, we discuss what mechanisms could drive such a configuration. First, stellar rotation of a single star is discarded as a likely cause. Second, the obvious interpretation of the double-wave photometry, i.e., a close binary system, is investigated. However unlikely, we discuss how the observed period change might be reconciled within a model of a strongly interacting binary. Third, an interpretation of a non-radial multi-mode pulsator is investigated. The observed period change and the multi-frequency behaviour do support this interpretation. We propose that the pulsational mode $l=1$ and $|m|=1$ may mimic a "binary" light- and radial-velocity curve. However, the phasing of the radial velocity and the light curve may be inconsistent. The possibility $l=2$ and $|m|=0$ is also discussed. Finally, we suggest how the enigma of the variability of WR 46 may be solved.
\end{abstract}

Key words. stars: Wolf-Rayet - stars: individual: WR 46 - stars: binaries: close - stars: variables: general stars: oscillations

\section{Introduction}

There is a long history of suggestions concerning the driving mechanism of the short-term (hours to days) cyclical variability of Wolf-Rayet (WR) stars. If a luminous companion was identified in the spectrum of a WR star and its radial motion appeared to vary in anti-phase with respect to the WR emission lines, the variability could unambiguously be attributed to binarity (Wilson 1939). However, in the absence of an observable companion no conclusive evidence can be provided in support of any of the suggested mechanisms: binarity, rotation and pulsation. The main reason for the ambiguity is that the source of the variability, i.e., the stellar core, is shielded by the optically thick

Send offprint requests to: A. M. van Genderen, e-mail: genderen@strw. leidenuniv.nl
WR wind. In addition, the instability of the wind may influence the effects of the variability of the core (Brown et al. 1995). A review of photometric and spectroscopic variability of WR stars in general is given by Antokhin (1996).

Various objects have been found to show short periods (days). They were suggested to be either close WR+O binaries (Massey \& Conti 1981), or to contain a compact companion (e.g., Moffat 1982). However, in some cases the periods proved not to be persistent, as in the case of, e.g., WR 103 (van Genderen \& van der Hucht 1986). Moreover, lack of strong X-ray emission was seen as evidence against the presence of a neutron star companion. Stevens \& Willis (1988) calculated that in the case of WR 6 (WN4) the $\mathrm{X}$-ray emission was much too low to expect a compact companion. Their calculation proved to be correct when 
it turned out that the luminous X-ray binary Cyg X-3 $\left(P_{\text {orb }}=4.8 \mathrm{~h}\right)$ contains a WR star (van Kerkwijk et al. 1992), possibly accompanied by a black hole (Schmutz et al. 1996; Hanson et al. 2000; though disputed by Mitra 1998).

Meanwhile, alternative explanations were put forward for the abovementioned short-term variability. For instance, in the case of WR 6 rotational modulation of a structured wind was suggested (e.g., St-Louis et al. 1995; Morel et al. 1998). Recently, however, the binary hypothesis for this best-studied example experienced a revival. Georgiev et al. (1999) showed that the object has a coherent phase diagram over a long time-span and suggested that the structured wind rotates $(P=3.77 \mathrm{~d})$ due to a companion. The same debate concerning binarity versus rotation was conducted by Morel et al. (1999) in favour of the latter for WR 134 (WN6), which shows a period of $2.25 \mathrm{~d}$.

Recently, in addition to WR 46, four new cases of persistent photometric periods of the order of a few hours have been found. If not due to a faint close companion in the diaphragm, the object WR 66 (WN8) shows a period of $P_{\text {phot }}=3.5 \mathrm{hr}$ (Antokhin et al. 1995; Rauw et al. 1996). Also the eruptive WR triple system in the Small Magellanic Cloud HD 5980 with an orbital period of $19.3 \mathrm{~d}$ shows a superimposed photometric period of $6.06 \mathrm{hr}$ (Sterken \& Breysacher 1997). The third object is WR 109 $(P=5 \mathrm{hr})$, which is also suggested to be a V Sge star (Cieslinski et al. 1999; Steiner et al. 1999). Also, according to Koenigsberger (2000, private comm.), WR 6 shows an additional $10.32 \mathrm{hr}$ period in the equivalent width $(E W)$, which remains to be confirmed. For these hourly periods of WR 66, WR 109, WR 6, and HD 5980 rotation is not a likely cause as the inferred rotational velocities are high relative to the break-up velocity. Although some of these objects are known binaries with periods of days, there is no evidence for any large radial-velocity variation on a time scale of hours. Therefore, multiplicity also does not seem to be a likely cause for the hourly periods. Thus, it is worthwhile investigating the possibility that the hourly variation is due to stellar pulsation.

Pulsational periods have been searched for by Bratschi \& Blecha (1996), but were not found in six selected WR stars. Those authors did find occasional variability for two objects during one night (out of many) with a quasi-period of 20-30 min. Besides, their data of WR 40 did not show the 10-min period discovered from earlier observations. It is unclear whether this intermittent behaviour is simply spurious or related to stellar pulsation, or to instability of the wind.

The following section presents a summary of the observational status of WR 46 as reported in Papers I and II. In Sect. 3, we discuss how the short-term cyclic variability can be viewed in general terms independent of a specific model. Subsequently, we discuss different models; singlestar rotation in Sect. 4, orbital modulation in Sect. 5, and single-star (non-)radial pulsation in Sect. 6. Section 7 presents the conclusions.

\section{Observational status of WR 46}

The nature of WR 46 is discussed extensively in Paper II, where it is inferred that the spectrum is formed in a stellar wind with optical depth larger than one. Moreover, the interstellar absorption line analysis by Crowther et al. (1995; henceforward CSH) indicates a distance of about $4.0 \mathrm{kpc}$. This is in agreement with membership of an OB association at $4.0 \mathrm{kpc}$ as suggested by ultraviolet photometry (Tovmassian et al. 1996; see also Veen \& Wieringa 2000). Hence, the object is luminous and we concluded that the light source of WR 46 is a Population I WR star, confirming the spectral analysis by CSH. The occurence of the O vi 3811-34 line complex, because of which the spectrum is annotated as "peculiar", is probably not due to an anomously high oxygen abundance, as suggested by CSH, but to, in particular, the susceptibility of that line doublet to low density (Paper II, Sect. 4.2).

\subsection{Summary of the short-term cyclical behaviour}

Within one night WR 46 often shows obvious photometric and spectroscopic variability. Here, we repeat the main observations as determined from our large photometric data sets and confirmed by smaller ones (Paper I), and from our simultaneous spectroscopic monitoring (Paper II):

1. it probably has multiple frequencies within one data set (observing epoch 1989-II shows $P_{\mathrm{sw}}^{89}=0.1412 \mathrm{~d}$ and $\left.P_{\mathrm{x}}=0.2304 \mathrm{~d}\right)$;

2. its colour turns red when optically bright and blue when optically faint, as expected for a hot star;

3. the frequency spectrum of the colour variability only reveals $f_{\mathrm{sw}}$, with its aliases and (sub-)harmonics;

4. the light curve probably shows a double-wave with unequal minima of on average a deep minimum of $\sim 0.04-0 .{ }^{\mathrm{m}} 07$ and a shallow minimum of $0 .{ }^{\mathrm{m}} 03-0 .{ }^{\mathrm{m}} 05$;

5. the colour-curve in 1989 indicates that the shallow minimum is bluer than the deep minimum, although the significance is not very high;

6. the line-flux variability mimics the continuum flux variability on the short-term time scale;

7. the line-flux variability has larger relative amplitude than the continuum flux variability;

8. the radial velocity often shows obvious variability and sometimes so-called stand-stills on the time scale of the photometric double-wave;

9. time-delays are observed from line-to-line in the case of (i) line-flux variability; even more so in the case of (ii) radial-velocity variability, and (iii) in the case of a start of a stand-still; and also (iv) within a single emission line, depending on the height above continuum. The higher part of an emission line is formed in the atmosphere, the larger the time-delay, which is in accordance with a time-lag of the outer layers with respect to inner layers of the stratified atmosphere;

10. He II 4686 shows, in addition to the line-flux and the radial-velocity variations, significant line-profile 
variability on a time scale of the same order as the identified periods.

Others have determined yet again another period. Niemela et al. (1995) found $P^{93 / 94}=0.31 \mathrm{~d}$ and Marchenko et al. (2000, henceforth MAB) found $P^{99}=0.329 \mathrm{~d}$ based on radial velocity measurements. MAB argue that these determinations are compatible. We suggest that the spectroscopy may not give a correct impression of the frequency of the variability. The radial velocity data are much more affected by time-delays (item 9ii) and seem to sometimes be completely out of phase. Therefore, radialvelocity studies may identify lower-frequency alias peaks, i.e., longer periods.

The variability of WR 46 is unique among the WR stars. None of the known short-periodic WR stars shows such large radial-velocity excursions, nor do any of the WR stars display an ellipsoidal light curve. In Sect. 3.1, we discuss how the observed variability relates to two well-studied objects. In Sect. 3.2 we discuss the possibility that the light variation is a single-wave and entirely disjoined from the radial motion of the line-forming regions. Subsequently, in Sect. 3.3, we assume a double-wave variation to be due to an ellipsoidally distorted wind.

\subsection{Summary of the long-term variations}

WR 46 turns out to vary also on a time scale of years, as first detected by the Hipparcos-satellite between 1989 and 1993 (Marchenko et al. 1998). Here, we summarize from Papers I and II the derived characteristics:

1. the long-term light variation results from the WR star, and not from a possible companion;

2. the long-term brightenings appear to be related to changes of the frequency which dominates the colour variations $f_{\mathrm{dw}}$. At least for one well-observed case (1989-1991), we find:

- a significant difference of $3.5 \%$ in $f_{\mathrm{dw}}$ occuring on the rising branch of the brightening. Although the brightness returned to its original level in the course of 1993, the 1995 data suggest that the frequency change persisted,

- the amplitude of the short-term variability increased by a factor 1.7 ,

- a slight reddening accompanied that brightening;

3. the short-term line-flux variability confirms the change of the photometric period change between 1989 and $1991 ;$

4. on the long-term time scale the line flux follows the photometric behaviour from faint to bright states;

5. other long-term brightening cycles up to 0. 3 appear to have occurred in 1998 and 2000, while spectroscopic evidence indicates brightenings before 1989 too;

6. there is a hint of a slow rise in $E W$ over the last fifty years.

We note that the long-term brightening of WR 46 is a unique feature in the Hipparcos survey of $67 \mathrm{WR}$ stars.
Furthermore, assuming that the long-term light curve is not sinusoidal but better described as a single event (possibly recurrent), we do not recognise the shape of the light curve as characteristic for a specific phenomenon, e.g., it does not look like S Dor- (=LBV-)pulsations, nor does it resemble the long-term behaviour of Super Soft Sources, or that of V Sge (Šimon \& Mattei 1999), a group of objects to which WR 46 erroneously has been suggested to belong (Paper II).

Since the terminal wind velocity seems unaffected and the line-widths are constant, it is concluded that the brightening is due to an increase in mass-loss rate. Moreover, application of a luminosity - radius relation based on stellar models indicates that the stellar radius increased by $4 \%$, and even more towards the peak of the brightening by the end of 1991 . We cannot ascertain whether the observed period and amplitude variations of the short-term variability are related to the brightening, but in the following discussions we will assume that this is the case.

\subsection{Flare-like variability}

In Paper II a flare-like behaviour was revealed in the spectroscopic monitoring data. In a total of 17 hours of monitoring, we observed a "bump" in two He II emission lines lasting at most $5 \mathrm{~min}$. Similar events may have been observed for other WR stars at least twice (Paper II). There is too little information to address the significance of this event for the interpretation of the periodic variability. Therefore, this phenomenon will not be addressed further.

\section{Discussion of the short-term cyclical variability}

\subsection{Comparison with WR 6 and $C y g X-3$}

Before we discuss models of WR 46, we compare it to the status of WR 6 (EZ CMa = HD 50896, WN4), whose periodic variation has been subject of much debate (e.g., Duijsens et al. 1996 and references therein). The radialvelocity variations are so small (and epoch dependent) that they can be considered as line-profile variations due to a rotating structured wind (Morel et al. 1997, 1998), in marked contrast to complete line-shifts of WR 46. During several epochs its light curve showed a double wave, like WR 46. However, during other observing runs, the light curve showed only one or even three (!) maxima, and sometimes none. Note that WR 46 sometimes shows deviating individual cycles, while its mean characteristic behaviour over many cycles does not change. Even when the light curve of WR 6 shows a double-wave, the phasing of the radial velocity does not agree with that of the rotation of a distorted star. This may not be surprising, since the period of WR 6 is roughly ten times larger than that of WR 46. Recently, Georgiev et al. (1999) confirmed WR 6 to have a coherent phase dependency with an improved period $(P=3.7650 \mathrm{~d})$ over more than 15 years, albeit 
with an epoch-dependent amplitude. Those authors consider this coherency to be indicative of a companion.

We also compare the photometric variability of WR46 with that of the short-period binary Cyg X-3 (WN4-7+c, $P=4.8 \mathrm{hr}$ ). It appears that its light curve, observed only in the infrared (because of a huge visual extinction) is a single-wave with its minimum coincident with the X-ray minimum. The single-wave is ascribed to the major influence of the X-rays on the stellar wind of the WR star (van Kerkwijk et al. 1996). The only long-term intense (non X-ray) photometric monitoring of this system was reported by Mason et al. (1976). Those authors showed that the light curve displayed a large variety of shapes. A few cycles were well-behaved and matched the X-ray light curve perfectly. Many other cycles are only reminiscent of the orbital variation, because they show a dip consistent with the timing of the X-ray minima. However, on at least two occasions the minimum is absent! Also, other investigators observed considerable variations of the light curve from night-to-night (Wagner et al. 1989), or from one season to another (Jones et al. 1994).

We conclude that Cyg X-3 displays a similar pattern of variation as WR 46 - from an accurate orbital modulation to constancy. Despite these changes of the variability, the frequency analysis by Mason and collaborators did produce significant peaks at the $4.8 \mathrm{hr}$ period and its harmonics. Thus, at least in the case of Cyg X-3, the genuine period (known from X-ray photometry) can indeed be identified amongst the intricate optical variability. This supports our interpretation that the different periods detected in WR 46 indicate indeed a true period change.

\subsection{Why (not) single-wave variability?}

Since the double-wave nature of the photometric variability is not beyond doubt (Paper I) and the radial velocity did not show a period consistently (Paper II), we need to address the possibility that the continuum, line flux and colour variability is controlled by the single-wave frequency $\left(P_{\mathrm{sw}}\right)$. In this scenario the radial-velocity period might be equal to, say, that determined by MAB $\left(P_{\mathrm{rv}}^{99}=0.329 \mathrm{~d}\right)$. Thus, a combination of mechanisms may explain the variability. The radial-velocity variation could be due to a different pulsational mode from that causing the photometric single wave, or, it could be due to binarity. Note that this implies that the radial-velocity and the line flux of the same emission line are controlled by different phenomena. However, as the variability is so enigmatic, we cannot exclude this possibility. Many more monitoring observations would be needed to constrain this scenario.

\subsection{Double-wave variability due to a rotating distorted wind}

In Paper II we gave a satisfying explanation of the trend of the colour versus brightness of WR 46 based on the WR Standard Model. When the star is hot (blue), more flux emerges in the EUV and the star becomes optically fainter. However, the outcome is not so satisfying in combination with the observations of the line fluxes. Calculations show that when the star is hot and, therefore, optically faint, the emission of the optical lines is expected to be stronger, while observations show the continuumand line fluxes to be in phase. Moreover, this interpretation ignores the effects of changes of the radius of the emission-forming layer. Therefore, and inspired by the ellipsoidal light curve, we investigated a geometrical interpretation of the variability.

Although the double-wave structure of the photometric variability is not proven (Paper $\mathrm{I}$ ), we assume in the following that it is the right interpretation. This view is supported by the unequal minima in 1989 and in 1991, the difference in the colour of the minima in 1989, and the length of the radial-velocity cycle. The double-wave light(and line-flux-) curves can be interpreted as being due to the rotation of an ellipsoidally distorted stellar wind, if we ignore the temperature dependence. Thereby, we also reach an alternative explanation of the "peculiar" colour behaviour. A schematic representation of the distorted wind is shown in Fig. 1 using the 1991 data. Below, we describe how we arrived at this representation, independent of the underlying source of distortion. Various mechanisms, which may invoke distortion, will be discussed in Sects. 4, 5, and 6 .

We determined (Paper II) the ratio of the maximum over the minimum flux during the ellipsoidal cycle. The projected surface during minimum light is proportional to $b^{2}$, and during maximum light to $a b$, where $a$ and $b$ are the long and short radius of the ellipsoid, respectively. Thus, the ratio of the maximum to minimum flux is a measure of the distortion $(a / b)$ of the line- or continuumforming layer. Table 2 in Paper II lists these ratios of each observing run ordered from the inner to the outer wind regions. The mean amplitude of the spectroscopic measurements is rather ill-defined because of the low number of observed cycles (2-3) per season. However, it can serve as a relative measure, since all lines are observed simultaneously. The line- and the continuum-flux scale, to the zero-th order, with the wind density (e.g., van Kerkwijk 1993); here we ignore the fact that the wind velocity is not constant. The table reveals a rising trend of the amplitude when going out in the WR atmosphere to the formation region of either He II 4859, or, in 1991, O VI 3811-34, and, subsequently, the ratios decrease when going further in the wind. The observed trend means that close to the star, the density distribution has an increasing ellipsoidal shape, while, further out, the wind continues to flow in a more spherical fashion. The peculiar colour behaviour (red when bright) is in line with the increasing distortion going outwards in the inner wind, i.e., the red continuum layer, formed outside the blue layer, is more distorted than the blue continuum layer; thus, the object appears redder when observing the ellipsoids on the side.

We apply the radii (in terms of stellar radii) resulting from the spectral fitting by CSH. We included the use of an 


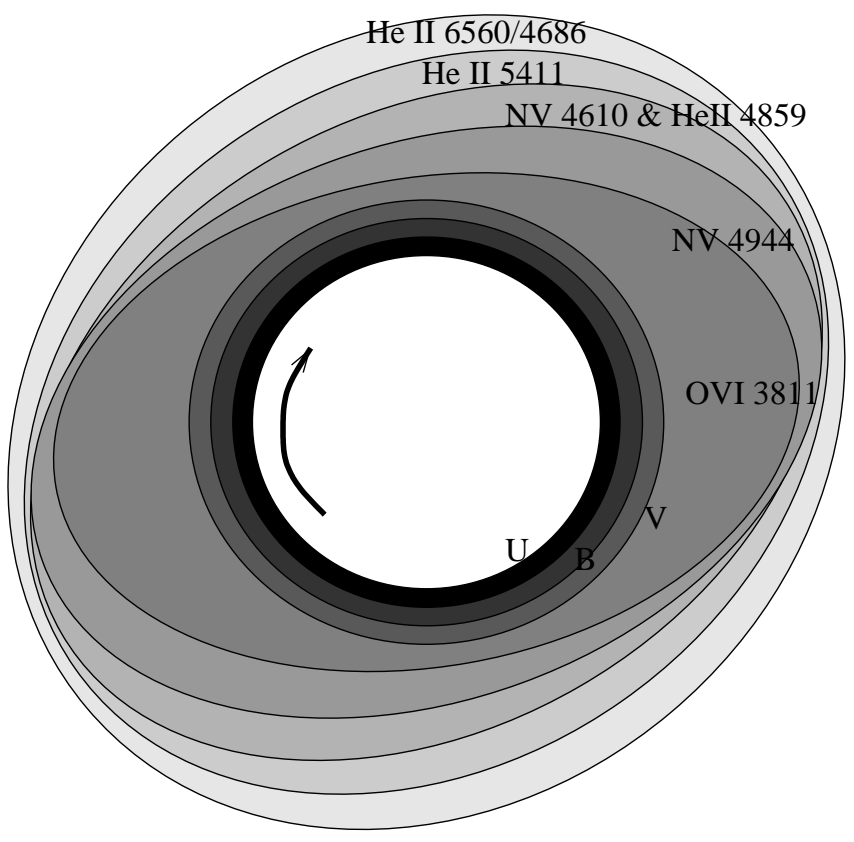

Fig. 1. A cartoon representation of the rotating distorted wind (pole-on view of WR 46), showing its stratification, based on the data from 1991. The observed amplitude of the continuum flux (Walraven $U, B$ and $V$ ) and line fluxes (as indicated) provide the ratio of the axes of the respective ellipsoids (turn the page 90 degrees to note the deformation). The actual distortion may be larger when an inclination angle $<90^{\circ}$ is taken into account. The orientation of their major axes depends on the measured mean phase-delay between the $E W$ curves of the spectral lines. The sizes of the ellipsoids are relative to the stellar core (white circle) as determined from the atmospheric model of CSH. The size of the stellar core might be much smaller, according to the stellar interior models (see text).

angle to represent the time-delay as measured in Table 3 of Paper II. The ultraviolet continuum is formed deeper in the atmosphere than the visual continuum (Schmutz 1997). The relative radii of the continuum-forming regions for the Walraven pass-bands are estimated, assuming that free-free emission dominates the production of photons. Assuming that the wind velocity increases linearly with the distance from the star and that the temperature is inversely proportional to the distance (polytropic wind structure with $\Gamma=3 / 2$ ), then the radius of the continuumforming layers becomes dependent on the wavelength as $R_{\lambda}\left(\tau=\frac{1}{3}\right) \propto \lambda^{2 / 3.5}$. Since $\lambda_{V}=5441 \AA$ and $\lambda_{U}=3630 \AA$, the two radii relate as $R_{V}=1.26 R_{U}$. Obviously, Fig. 1 is an idealized "picture" of WR 46.

\subsubsection{Mass-loss flux varying over the stellar surface}

In the discussion above, the short-term variability is interpreted as being due to a rotating ellipsoidal density distribution of an optically thick stellar wind. The density changes in the wind appear not to be related to a varying velocity field, as the widths at zero intensity of the various emission lines remain constant, while the line-intensity changes. This means that enhanced mass-flux occurs in the wind along the major axis of the ellipsoids. Either the mass-loss is spherically symmetric and the flow is bent towards the major axis, or, the mass-loss is aspherical, being larger along the major axes at the stellar surface. Since (i) any disturbance (companion, pulsation etc.) of the physical conditions below the sonic point affects the mass-loss rate (Lamers \& Cassinelli 1999) and, (ii) bending the wind requires large magnetic and/or radiative forces, we conclude that the mass-flux varies over the stellar surface.

\section{Single-star rotation}

As mentioned in Sect. 1, in general, stellar rotation of a single WR star is not a likely cause of short-term variability with a period much shorter than a day. The evolved nature of the WR star and its large mass-loss rate strongly disagree with a rapidly rotating single star. Note that the bipolar structure of the wind is indicative of a bipolar magnetic field, which would argue more strongly against rapid rotation. Without a magnetic field the photosphere of a rapidly rotating WR star becomes disk-like (Langer \& Heger 1999).

In the case of WR 46, the long-term behaviour enhances the problems for the single-star rotation model. In the case of rotation the period decrease (Paper I) implies a stellar radius decrease. Because the star brightens, the temperature has to increase significantly, while the spectra do not support such a change. We conclude that the observations cannot be reconciled with single-star rotation.

\section{Double-star model}

\subsection{The double-wave variability}

In Sect. 4.3 .3 we propose that variability is due to the rotation of a distorted wind and star. The distortion of the star (and the wind) may have been induced by a companion. Normally, an ellipsoidal light curve is interpreted in the framework of Roche geometry (Fig. 2). For a normal ellipsoidal curve, the star is hot (blue), when looking on its side, and bright due to its temperature and larger area, as presented in the upper half of Fig. 2 .

The bottom half corresponds to the observations of WR 46 with the peculiar colours and the interchange of the deep and the shallow minimum. We note that the deviation from "normal" ellipsoidal behaviour cannot be attributed to the light from a companion. Either the companion is bluer (redder), which will cause blueing (reddening) when it is in front, and vice versa when behind. Thus, a double wave in the colours is not possible. Moreover, the surface brightness of the companion should be larger than that of the WR star because of the deep minimum, while the integrated light has to be only of the order of a percent of the WR luminosity. This is unlikely for any type of companion.

With the distorted-atmosphere model developed in Sect. 3.3, we interprete the observations as follows. The spheroid of the red-continuum forming layer is further 


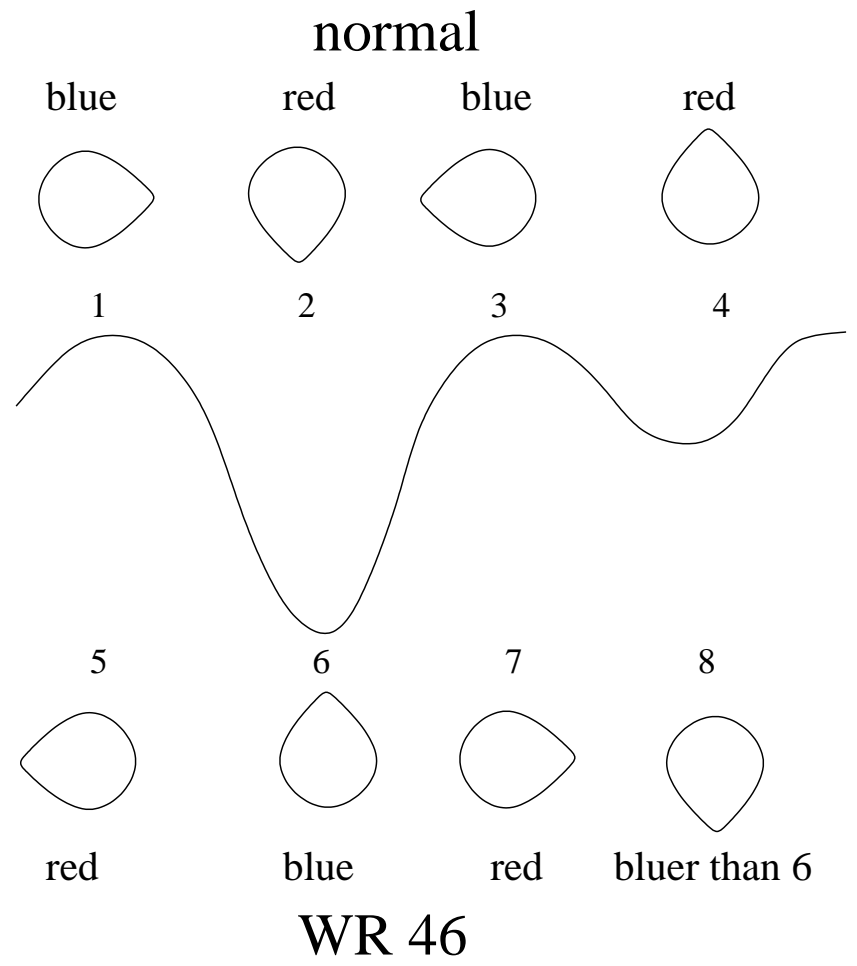

Fig. 2. Schematic representation of a rotating distorted star with its point-shaped part facing a companion. The observer is looking from the bottom side of the picture. Upper part: standard ellipsoidal light variation. Lower part: our interpretation of WR 46 (Sect. 3.3) where the light minima and the colour behaviour is contrary to normal ellipsoidal behaviour (see Sect. 5.2).

away than the blue-continuum forming layer. Since the distortion increases going outwards, the ratio of the red to the blue spheroid is larger when looking sideways than when looking on its end points. Thus, when bright (looking sideways) the star appears redder.

When looking on its side the star is bright because of the large area. And as the distortion is larger for redcontinuum forming layers further from the star, the star appears redder when viewed sideways!

\subsection{Constraints on the binary parameters}

Assuming that the radial-velocity variation of the emission lines reflects the orbital velocity in a binary system, we derive constraints on the system parameters. The amplitude of the radial-velocity variation of the N V 4603/19 line is much larger than that of all the other lines, as was also found by Niemela et al. (1995). This line complex is much more sensitive to changes in the stellar parameters (see Paper II Sect. 5.2). We assume that the projected orbital velocity is best represented by the inner wind lines ( N v 4945, O VI 5290), and we will use a value of $K=v \sin i=75 \mathrm{~km} \mathrm{~s}^{-1}$ (Paper II Sect. 3.2). For the case in which the amplitude is higher, we refer to MAB who discuss $K=90 \mathrm{kms}^{-1}$. MAB note that change of the
$R V$ amplitude may be linked to secular flux variations. Consequently, they reach very different results.

\subsubsection{Mass-function and inclination}

We derive a mass function $f\left(M_{2}\right)=0.012 M_{\odot}$. This mass function is six times larger than the value derived by van Genderen et al. (1991), because they measured the radial velocity by folding the spectral lines with a Gaussian reducing the amplitude to $K=40 \mathrm{~km} \mathrm{~s}^{-1}$. Niemela et al. (1995) even found much larger values of 0.17 and $1.06 M_{\odot}$ using, respectively, the $\mathrm{N} \mathrm{v} \mathrm{4603/19} \mathrm{and} \mathrm{He} \mathrm{II} 4686$ lines, which we consider inappropriate tracers of the orbit. MAB use the same tracers as we do and their $K$-amplitude results in a value of $0.02 M_{\odot}$. Our value of the mass function is relatively small and points to a low-mass companion, and/or an extreme mass ratio, and/or a low inclination angle (polar like). Also the absence of eclipses indicates that the inclination angle cannot be very large, unless the companion is a small degenerate object (WD). We estimate the inclination angle to have an upper limit of $60^{\circ}$ for a non-degenerate companion.

\subsubsection{Possible type of companion}

$\mathrm{CSH}$ derive a X-ray luminosity of $5 \pm 2 \times 10^{31} \mathrm{ergs}^{-1}$, which is typical of single WN stars. Despite the lack of copious X-rays, MAB discuss the possibility that the companion is a degenerate companion. Various mechanisms (e.g. for rotational inhibition see Stella et al. 1986, for optically thick accretion disk see Pollock 1995) have been proposed to explain the lack of X-rays in such a case. However, the finding that Cyg X-3 (Sect. 3.1) contains a WR star provides strong indication that the companion of WR 46 is not compact. In the following we wish to discuss other types of companions, which have received less attention in the literature. The X-ray luminosity cannot exclude a hydrogen main sequence (MS) star, a helium star (He-star), or a white dwarf (WD) as a companion.

Assuming that WR 46 is a binary, Fig. 3 illustrates the constraints inferred for the binary parameters using the Roche-lobe geometry. As an indication, it presents the solutions for three different values of the inclination: $20^{\circ}$, $40^{\circ}$, and $60^{\circ}$, using $K=75 \mathrm{~km} \mathrm{~s}^{-1}$. The top right panel in Fig. 3 shows that the maximum radius of the companion (= secondary) is independent of the inclination angle, but depends solely on its mass. Obviously, a massive nondegenerate star does not fit into the orbit. Using a crude mass-radius relation for MS objects $\left(M\left(M_{\odot}\right)=R\left(R_{\odot}\right)\right)$, it appears that such a companion needs to be less massive than $0.75 M_{\odot}$. Such a star would be rotating at $28 \%$ of its critical velocity, when it is in co-rotation. Possibly, the mass is somewhat lower due to the centrifugal and gravitational potential (Huang 1999) and radiation pressure (Drechsel et al. 1995).

We also indicated the He-burning MS in the panel as determined by Langer (1989). The formula given by 


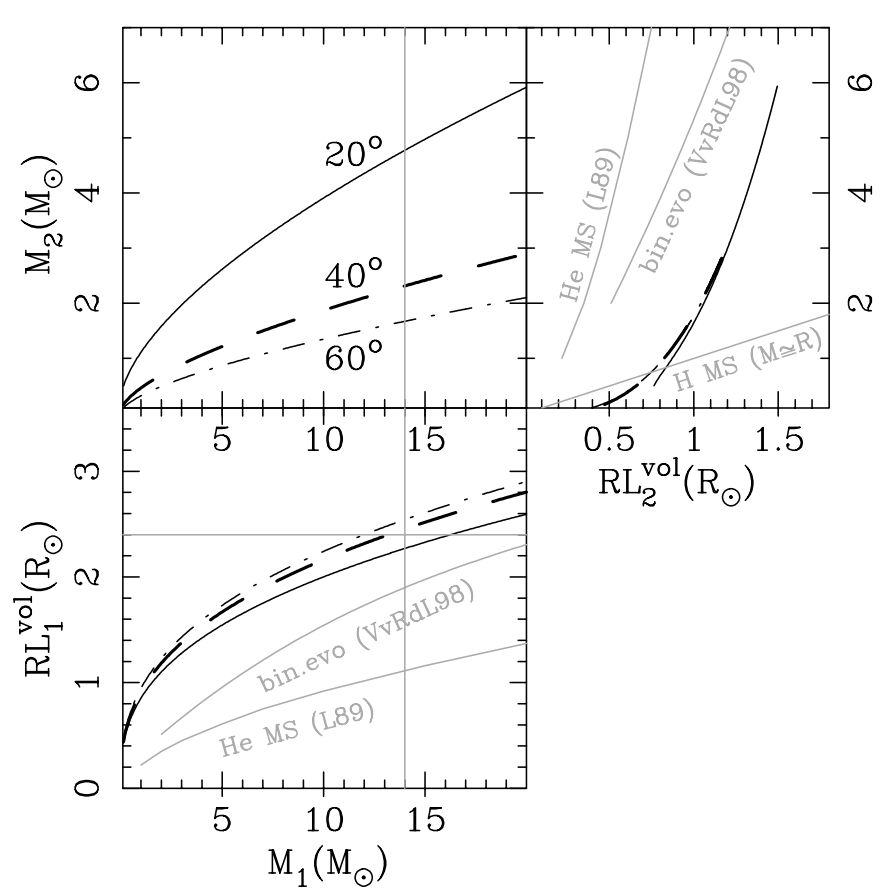

Fig. 3. Constraints on the masses of possible binary components of WR 46. In each panel the solution for $i=20^{\circ}$ (full line), $i=40^{\circ}$ (dashed), and $i=60^{\circ}$ (dash-dotted) is shown. Upper left: the primary mass - secondary mass relation using $K=75 \mathrm{~km} \mathrm{~s}^{-1}$. Upper right: Roche-lobe (volume) radii of the secondary $\left(R L_{2}\right)$; the mass-radius relations for $\mathrm{H}-$ and $\mathrm{He}-$ burning MS stars are indicated in grey (L89 = Langer 1989); also shown is the mass-radius relation for hydrogen-stripped stars on the onset of He-burning (VvRdL98 = Vanbeveren et al. 1998: their Eq. (12.4)). Lower panel: Roche-lobe radius of the primary WR-star $\left(R L_{1}\right)$ versus its mass; the core-radius of the WR star as determined from atmosphere models (CSH; $\mathrm{HK}$ ) and its mass from a mass-luminosity relation are indicated $\left(2.4 R_{\odot}, 14 M_{\odot}\right)$.

Schaerer \& Maeder (1992) differs only marginally. Clearly, a low-mass He-star would fit within this Roche lobe. Since the mean spectrum and behaviour of WR 46 is dominated by one WR star, we presume that a possible He-star companion should have a much lower luminosity, and, therefore, be of lower mass. Of course, the Roche-radius does not restrict the option of a WD companion.

From an evolutionary point of view the last option requires the most peculiar scheme and a He-star also demands some fine-tuning, but neither is impossible per se (see also Portegies Zwart \& Verbunt 1996). As for a nondegenerate companion, Vanbeveren et al. (1998) predicts that $3-5 \%$ of the WR stars have an intermediate or lowmass companion. It is understandable that such companions would stay unnoticed, unless the binary is strongly interacting, as may be the case for WR 46.

\subsubsection{The WR component}

If the WR stellar core would be as large as determined from spectral analyses, i.e., $2.4 R_{\odot}(\mathrm{O}-\mathrm{He}$ model by $\mathrm{CSH})$, it would be (close to) filling its Roche lobe, as can be

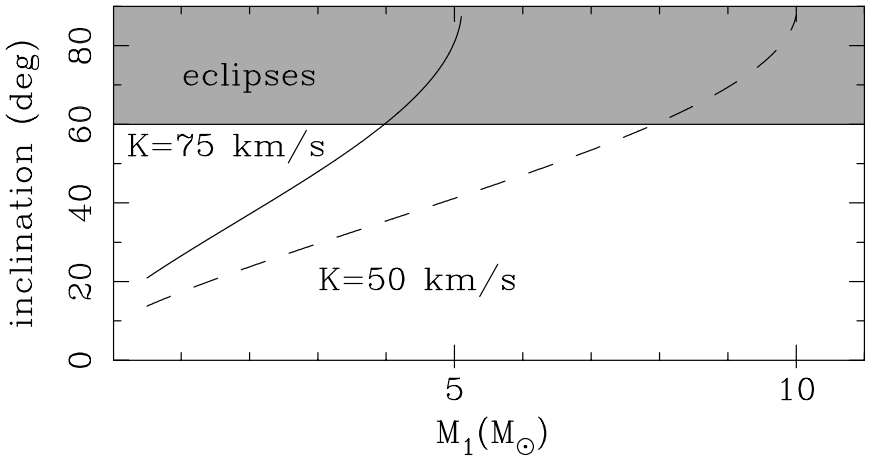

Fig. 4. Possible mass of WR 46, assuming that the secondary is a MS star filling its Roche lobe of $M_{2}=0.75 M_{\odot}$. The lines (solid: $K=75 \mathrm{~km} \mathrm{~s}^{-1}$ and dashed: $K=50 \mathrm{~km} \mathrm{~s}^{-1}$ ) indicate the constraints on the mass of WR 46 . We roughly indicated at what inclination eclipses could be expected.

observed in Fig. 3 (bottom panel). However, there is considerable evidence that spectral analyses overestimate the stellar radius (Moffat \& Marchenko 1996; see also van Kerkwijk et al. 1996), because it extrapolates the velocity law. The inaccuracy of the standard velocity law is also evident from observations of WR 46 itself (Paper II). According to the modelling of He-cores by Langer (1989) and Vanbeveren et al. (1998), the WR stellar radii are much smaller and WR 46 fits easily within its Roche lobe.

If the companion is a massive white dwarf of about $1.3 M_{\odot}$, or a low-mass low-luminosity He-star of $1-3 M_{\odot}$, the WR stellar mass is in the range of $10-15 M_{\odot}$ (Fig. 3). On the other hand, if the companion is a Roche-lobe filling MS star of much lower mass $\left(0.75 M_{\odot}\right)$, the WR star needs to be less than 5 , or even $4 M_{\odot}$ (see Fig. 4). Using a mass-luminosity relation (Langer 1989; Schaerer \& Maeder 1992), CSH determined a mass of $14 M_{\odot}$. However, a low mass cannot be excluded for WR 46, because of the uncertainty in the distance and the effects of rapid rotation (Meynet 1998; Heger et al. 2000; Heger \& Langer 2000). Table 1 lists four different options for the binary hypothesis of WR 46.

\subsection{Period change not due to orbital decay}

The large difference in period between the Walraven data in March 1989 and in February 1991 of WR 46 may not be a true period change. For instance, the period in 1989 and the one in 1991 may not reflect the same phenomenon. Say, binary modulation may dominate in one season, while stellar pulsation grows to dominate the light curve in the other season.

In this respect, it is noteworthy that the period change is reminiscent of the so-called (negative) superhump phenomenon (e.g., Patterson et al. 1997) in cataclysmic variables, i.e., an accretion disk during outburst can show a precessing high-density ring with a precessing period a few percent larger (smaller) than the orbital period. However, except for the amplitude and period, the phenomenology of the short-term variability of WR 46 does not show 
evidence of the occurence of a new phenomemon, and certainly not related to a disk, for which we do not see any evidence in the first place. Moreover, although the period and amplitude changed, the observed cycles show much resemblance and same colour behaviour and unequal minima in 1989 and 1991. Thus, the period change is not easily reconciled with the occurence of a new phenomenon, e.g., a superhump.

\subsection{Period change due to orbital decay}

The difference of the period between 1991 or 1989 and the period detected by MAB is so large that it cannot be reconciled as a true change of an orbital period. In this subsection we only consider the period change between 1989 and 1991. The proposition at this point is that the period as detected by MAB does not represent the orbital period. Note that the period in 1999 (MAB) is based on spectroscopy, which might be hampered by the radialvelocity stand-stills.

\subsubsection{Energy and angular momentum budget}

First we investigate the conservation of angular momentum and of energy. Table 1 also presents the spin energy $\left(E_{\text {spin }}\right)$ and angular spin momentum $\left(J_{\text {spin }}\right)$ of the primary WR star, and the orbital energy $\left(E_{\text {orb }}\right)$ and orbital angular momentum $\left(J_{\text {orb }}\right)$ of four binary configurations, assuming co-rotation. The basic formulae are listed in Table 2. The quantities representing the spin depend on the moment of inertia $\left(I_{1}\right)$ of the WR star (the amount of spin of the companion can be ignored). The moment of inertia depends on the density distribution, i.e., the geometry parameter $\eta$. For a solid sphere $\eta=0.4$, and $\eta<0.4$ for objects more densely concentrated, like stars. We received, prior to publication, recent WR stellar models by Meynet \& Maeder (2000). For a $12 M_{\odot}$ WC star, $\eta$ turns out to be 0.04 , while for a $30 M_{\odot}$ WN star with still some hydrogen $\eta$ is about 0.08 . We estimate that the structure of an early-type WN star will be in-between these values and, therefore, we use a value for WR 46 of $\eta=0.06$. Note that this estimate is based on single-star models.

The last column in Table 1 shows the relative changes inferred from the observed period change of $-3.5 \%$, assuming orbital decay without mass-loss or -transfer. The changes of $J_{\text {spin,1 }}$ and $E_{\text {spin,1 }}$ do not include the possible radius increase of $4 \%$. Therefore, these variations are listed as lower limits. From Table 1 we can easily make up the energy and angular momentum budgets. For example in case I (rather massive He-star companion) the orbital angular momentum (1.75e53) decreases by $1.1 \%$ due to the period decrease, while the spin angular momentum (1.2e52) increases by $3.5 \%$ assuming corotation. Evidently, the loss of orbital angular momentum is four to five times larger than the WR spin can absorb. This problem is even larger when a more realistic radius of the WR star is assumed (cases II and III). Only in case IV,
Table 1. Four conceivable binary models which may represent WR 46, at least with respect to the short-term variability. Three cases have $K=75 \mathrm{~km} \mathrm{~s}^{-1}$ and one has $K=50 \mathrm{~km} \mathrm{~s}^{-1}$, to emphasize the uncertainty of the true $K$-amplitude $(K$ amplitudes higher than $75 \mathrm{~km} \mathrm{~s}^{-1}$ can certainly not accomodate the large orbital change). The last column lists the percentual change assuming that the period change indicates orbital decay. The list of cases shown here is by no means complete. The primary is identified with the WR component. $M, L, R, a$ and $R L$ (=volume Roche-lobe radius) are in solar units, while the orbital frequency $\omega$, the moment of inertia $I$, the angular momenta $J$, and the energy $E$ are derived using the formulae in Table 2 in cgs units.

\begin{tabular}{lrrrrr}
\hline \multicolumn{1}{c}{ case } & \multicolumn{1}{c}{ I } & \multicolumn{1}{c}{ II } & III & IV & \\
comp. & He-MS & H-MS & WD & H-MS & d/d $t$ \\
\hline$K$ & 75 & 50 & 75 & 75 & \\
$M_{1}$ & 14 & 10 & 5 & 7 & \\
$M_{2}$ & 4 & 0.7 & 0.7 & 0.7 & \\
$R_{1}$ & 2.4 & 1 & 1 & 2.4 & \\
$R L_{1}^{\text {vol }}$ & 2.32 & 2.40 & & & \\
$R_{2}$ & 0.5 & 0.7 & 0.7 & 0.7 & \\
$R L_{2}^{\text {vol }}$ & 1.31 & 0.74 & & & \\
$q$ & 0.286 & 0.07 & 0.14 & 0.10 & \\
$i$ & $18^{\circ}$ & $78^{\circ}$ & $68^{\circ}$ & & \\
$a$ & 4.73 & 3.97 & 3.22 & 3.67 & $-2.3 \%$ \\
$a_{1}$ & 1.35 & 0.28 & 0.45 & & \\
$a_{2}$ & 3.38 & 3.69 & 2.77 & & \\
$\omega$ & $2.57 \mathrm{e}-4$ & & & & $+3.5 \%$ \\
$I_{1}$ & $4.7 \mathrm{e} 55$ & $5.8 \mathrm{e} 54$ & $2.9 \mathrm{e} 54$ & $2.3 \mathrm{e} 55$ & $(+8 \%)$ \\
$J_{\text {spin, } 1}$ & $1.2 \mathrm{e} 52$ & $1.5 \mathrm{e} 51$ & $7.5 \mathrm{e} 50$ & $6.0 \mathrm{e} 51$ & $>3.5 \%$ \\
$J_{\text {orb }}$ & $1.75 \mathrm{e} 53$ & $2.60 \mathrm{e} 52$ & $1.6 \mathrm{e} 52$ & $2.1 \mathrm{e} 52$ & $-1.1 \%$ \\
$E_{\text {spin }, 1}$ & $1.5 \mathrm{e} 48$ & $1.9 \mathrm{e} 47$ & $9.7 \mathrm{e} 46$ & $7.7 \mathrm{e} 47$ & $>+7 \%$ \\
$E_{\text {orb }}$ & $2.3 \mathrm{e} 49$ & $3.38 \mathrm{e} 48$ & $2.1 \mathrm{e} 48$ & $2.6 \mathrm{e} 48$ & $-2.3 \%$ \\
\hline & & & & &
\end{tabular}

with a low-mass companion and a large WR radius, is the change of angular momenta of the same order of magnitude. In fact, this is the so called Darwin instability (e.g., see Kelley et al. 1983 about Cen X-3). The energy is also more or less conserved. Especially so, if one notes that the energy budget also involves the extra luminosity $E_{\mathrm{XL}}$ emitted during 2.5 yrs which amounts to about $10^{46} \mathrm{erg}$, and the energy corresponding to the increase of the massloss rate which amounts to $10^{45}$ erg. Below, we discuss mechanisms which might play a role in this respect.

Note that the $K$-amplitude by MAB is too large to reconcile with the rapid orbital decay because in that case the orbital energy and angular momentum are to large to be absorbed by the WR star.

\subsubsection{Tides causing brightening and orbital decay}

If tidal forces play an important role, the long-term brightening $\left(E_{\mathrm{XL}}\right)$ might equal the dissipation energy $\left(E_{\mathrm{dis}}\right)$ of 
Table 2. Formulae used in the analysis in Sect. 5.4.1.

\begin{tabular}{ll}
\hline$f\left(M_{2}\right)=\frac{P K_{1}^{3}}{2 \pi G}=M_{2}\left(\frac{q}{1+q}\right)^{2} \sin ^{3} i$ & $M_{\odot}$ \\
$I=\eta M R^{2}$ & $\mathrm{~g} \mathrm{~cm}{ }^{2}$ \\
$E_{\text {orb }}=-G \frac{M_{1} M_{2}}{2 a}$ & erg \\
$E_{\text {spin }}=\frac{1}{2} I\left(\frac{2 \pi}{P}\right)^{2}$ & erg \\
$J_{\text {orb }}=M_{1} M_{2}\left(\frac{G a}{M_{1}+M_{2}}\right)^{1 / 2}$ & erg s \\
$J_{\text {spin }}=\omega I=\omega \eta M R^{2}$ & erg s \\
\hline
\end{tabular}

the tidal bulges on the WR star. The torque $(\tau)$ exerted on the WR star by the companion can be calculated using:

$\frac{\mathrm{d} E_{\mathrm{dis}}}{\mathrm{d} t}=\omega \tau=1 \times 10^{46} \mathrm{erg} / 2.5 \mathrm{yr}$,

where $\omega$ is the orbital frequency. Since the torque is equal to the rate of angular momentum transfer, it follows that the absolute total transfer of orbital- into spin angular momentum by tidal bulges equals $\mathrm{d} J=4 \times 10^{49} \mathrm{erg}$ s. Table 2 shows that this amount is only a quarter, or less, of the angular momentum extracted from the orbit. However, it can explain the spin-up of the WR star with a small radius (cases II and III). Thus, although tidal dissipation might explain the long-term brightening, it can only explain a (small) part of the period decrease.

\subsubsection{Mass-loss causing brightening and orbital decay}

We investigate the assumption in Sect. 5.4.1 that the mass-transfer and -loss are ignored. Inspection of the formulae in Table 2 tells us that (i) conservative masstransfer from the WR star to the secondary enhances the energy "problem", i.e., the amount of orbital energy release becomes larger, and (ii) mass-transfer to the WR star enhances the orbital angular momentum "problem". Thus, only mass-loss from the system may help orbital decay.

Excessive mass-loss can only occur through the Langrangean points $L_{1}$ and $L_{2}$. In this respect, it is notewothy that the Roche-lobes may be severely affected by the radiation pressure from the WR star. Drechsel et al. (1995) have modelled the effects of radiative forces on the Roche-lobe geometry of a close binary system. They find that a luminous star (like a WR star) may "push" its companion from contact with the inner Lagrangean point $\left(L_{1}\right)$ to outer contact. Vanbeveren et al. (1998, their formulae 10.10 through 10.15) considered the effect of enhanced mass loss through the second Lagrangean point $\left(L_{2}\right)$. To explain the large period decrease, a mass-loss rate of order $10^{-2} M_{\odot} / \mathrm{yr}$ is inferred. However, this is probably much too large to stay unnoticed in the WR stellar wind. We conclude that modification of the Roche potential by radiation pressure may well be a very important ingredient to bring about peculiar effects, but it seems impossible to account for the extreme period change.

\subsubsection{Pulsational resonance causing orbital decay}

Pulsational resonance occurs if, due to orbital evolution, the orbital period approaches an eigen frequency of the star. During resonance a lot of momentum and energy is exchanged, causing rapid orbital decay. Most efficient in this respect would be the excitation of a low- $l$ gravitational mode, which happens to be consistent with the the size of the period (Sect. 4.6.1). The typical time scale for the development of such a resonance is

$T=\frac{1}{\Delta \sigma \Omega_{\mathrm{c}}}$

where $\Delta \sigma$ is the width of the resonance peak in frequency space, and $\Omega_{\mathrm{c}}$ is de critical rotation rate. Witte \& Savonije (1999) calculated the resonances of a $10 M_{\odot}$ MS star $(l=2$, and $|m|=2$ ) and found $\Delta \sigma$ to be in the range $10^{-3}$ (low $k$ ) to $10^{-6}$ (high $k$ ). Using these numbers for the WR star, we find a time scale in a range from a few weeks to 20 years. Although it remains rather speculative, it seems possible that the behaviour of WR 46 can be interpreted by pulsational resonance within a binary system.

\subsection{Discussion of the double-star models}

If we would not have detected a large period change, the short-term variability of WR 46 would undoubtedly be attributed to a low-mass, low-luminosity, probably non-compact companion. However, during a long-term brightening a different period is observed. Except for the different period and the somewhat larger amplitude, the variability shows the same characteristics, indicating it is the same phenomenon, i.e., a true change of period.

To illustrate how enormously large this change would be in case of an orbital period, we compare WR 46 to the most rapidly decaying binary SV Cen. This object is a massive contact binary $\mathrm{B} 1 \mathrm{~V}+\mathrm{B} 6.5 \mathrm{III}$ with a period $P=1.66 \mathrm{~d}$, showing on average $\dot{P} / P=1.52 \times t 10^{-5} \mathrm{yr}^{-1}$ (Drechsel et al. 1982), while the observed period change of WR 46 is $10^{3}$ times larger. However, the rate of period decrease of SV Cen is not constant, and can show sudden jumps up to $\dot{P} / P \geq 10^{-4} \mathrm{yr}^{-1}$, i.e., a period change of 15-30 s in two years (Herczeg \& Drechsel 1985; Drechsel \& Lorenz 1993). Also other binaries are shown to undergo irregular period variations (Herczeg 1992).

The mechanism for the orbital decay of SV Cen may be related to the large mutual radiation pressure of the luminous B stars (Drechsel et al. 1995). As the luminosity of WR 46 is 10 to 30 times larger and the mass ratio is more extreme, the effects of this mechanism may be more severe. Whether it suffices to enlarge the orbital decay by a factor of $10^{2}$ relative to the period jumps of SV Cen remains to be investigated.

The conservation of energy and angular momentum leads us to suggest that the WR star has a large moment of inertia, and is, therefore, extended and fills its Rochelobe. In turn, if the brightening equals the dissipation energy of the tidal bulges to spin up the WR star, it appears 
that the WR radius is as small as $1 R_{\odot}$. Additional mechanisms which may help to transport and/or remove angular momentum and energy are excessive mass-loss from the system through the Lagrangian points, and pulsational resonance. In conclusion, considering the conservational laws and various mechanisms it is not excluded that the observed period change from 1989 to 1991 represents an orbital period change as in the case of spiral-in. However, we consider it highly unlikely. In comparison to the 1989 and 1991 period it is even impossible to accept the period by MAB as the orbital period in 1999 .

\section{Single-star (non-)radial pulsation}

Radial pulsations are characterised by the number of nodes along the radius of the star $k$. Non-radial pulsations are characterised by the numbers $l$, indicating the total number of nodal lines (circles) over the stellar surface, and $m$, the number of nodal lines crossing the equator (great circles). We note that so-called sectoral modes may occur $(l=|m|)$, zonal modes $(m=0)$, or tesseral modes $(l>|m|, m \neq 0)$.

Since the variability would be averaged out in the photometry if the number of nodal lines would be large, the number of nodes needs to be small. It turns out that for WR 46, when its mass and radius are compared with those of slowly-pulsating B-stars, a low-l gravitational mode may indeed have a period of a fraction of a day. In contrast, the periods of radial pulsations are predicted to be smaller than one hour for WR stars (see also Sect. 6.4).

\subsection{The double-wave frequency due to pulsation}

Since radial pulsations cannot cause a photometric doublewave, the question is whether a low-order non-radial mode could invoke a distortion of the wind, which appears to rotate as in Fig. 1. We propose that this is possible with the lowest order sectoral mode $l=1$ and $|m|=1$, which results in a single bright hemisphere travelling along the equator. Figure 5 presents the schematic picture we have in mind. Presumably, the bright hemisphere could distort the wind to cause an asymmetric density flow. As the pulsational pattern travels over the (invisible) stellar surface, the "one-armed" wind will appear to rotate as well. Thus, when the bright hemisphere points at right angles to the line-of-sight (twice during one period), the projected surface of the ellipsoidal wind would be largest, causing a photometric double-wave during a single pulsational period. In this model the star is decentered relative to the ellipsoids causing the endpoints to differ in line with the difference of the photometric minima.

Moreover, since the enhanced outflow from the bright hemisphere points towards the observer once per cycle, the radial velocity is indeed modulated with a single wave. The phasing, however, does seem to be inconsistent. Maximum light should occur when the velocity passes the equilibrium velocity, while our observations indicate that light maxima occur during maximum red shift- and blue shift velocity.

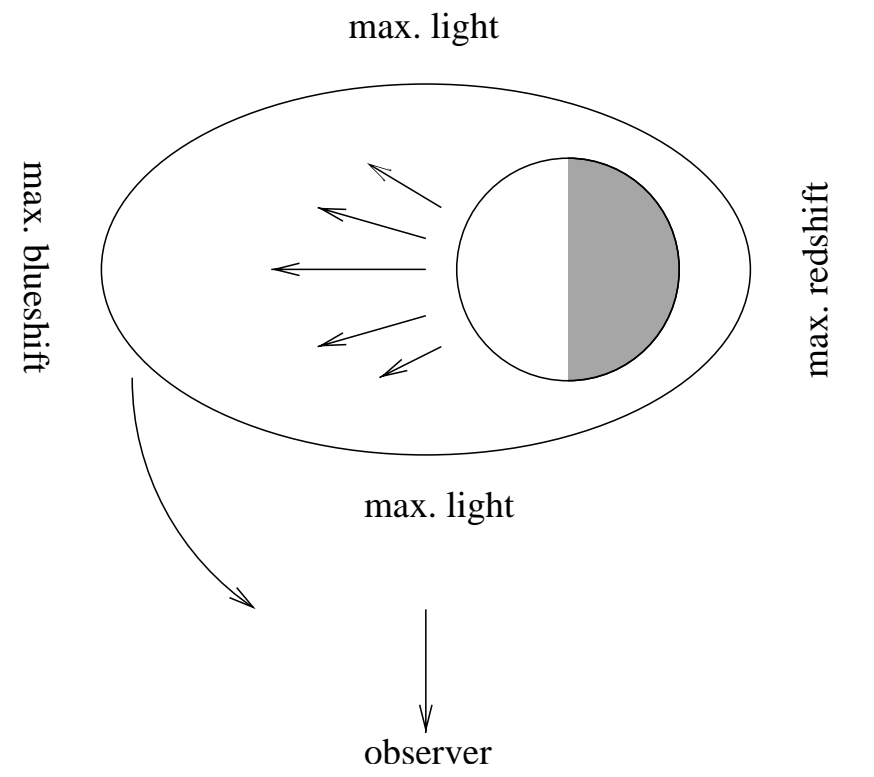

Fig. 5. The hypothesized schematic appearance of an $l=1$ $|m|=1$ pulsational mode in case of a WR star (top view). The bright hemisphere (white) produces enhanced outflow causing the distortion of the visible surface (ellipsoid), where $\tau=1 / 3$. As the pulsational pattern travels over the invisible surface, the distorted density distribution rotates as indicated.

Note that this is also evident when considering the radialvelocity and the line-flux curves of the same line as well in our data as in data by MAB However, counter examples are observed, and we are suffering from a small number of cycles covered by the observations. Moreover, the large time-delays of the radial velocity disturb the simple picture sketched above.

Alternatively, the star could show a zonal mode with $l=2$ and $m=0$ observed pole-on. A bright pole pointing towards the observer would cause a light maximum. A bright equator causes enhanced outflow in the plane of the sky, which may enlarge the projected area of the wind and induce a second light maximum during a single pulsation. The blueward radial velocity may be maximum when the pole is bright causing a single wave radial velocity modulation. Note, however, that in this model-configuration the maxima are different while the observations show the minima to differ. In addition, since both poles are bright simultaneously, we have to assume that the enhanced redward velocity is hidden from our sight. In summary, it seems possible that pulsation of a WR star may mimic a binary light- and radial-velocity curve.

\subsection{The single-wave frequency $\left(P_{\mathrm{sw}}\right)$ and the additional frequency $\left(P_{\mathrm{x}}\right)$ due to pulsation}

In continuation of the discussion in Sect. 3.2 that the radial-velocity variability is not related to the photometric variability. In that case, the radial velocity may indicate that WR 46 is a binary system, while the photometry may result from a pulsating WR component. We point out that such pulsational periods in addition to the main 
periods are found in other WN single- and binary stars, i.e., WR 66, HD 5980, and WR 6 (see Sect. 1). These stars have been monitored spectroscopically so intensively, that we can safely assume that large radial-velocity excursions on time scales of the additional frequencies did not escape attention. These pulsations are considered to be nonradial, but the pulsational mode cannot be identified.

The additional period of WR 46 in 1989, $P_{\mathrm{x}}$ does not appear in the colour variability, it seems not to be related to large radial-velocity variations, and it is not observed in other seasons. Therefore, independent of the interpretation of the single- or double-wave variability, the additional period $\left(P_{\mathrm{x}}\right)$ can be interpreted as due to pulsation. We hypothesize that the absence of any colour effect indicates a different type of pulsational mode, i.e., a sectoral, zonal, or tesseral mode, than is inferred for the main variability.

\subsection{Long-term changes in the case of pulsation}

We address the interpretation of the period change in the case of stellar pulsation. Since the appearance of the cycles in 1989 and in 1991 is rather similar, we first assume that the $l$ and $m$ of the pulsational mode do not change. In that case the period decrease can be understood if the radius of the star decreased. However, we have argued in Sect. 4 that this is unlikely because of the brightening. Alternatively, a change of the number of radial nodes $k$ may explain the observed period decrease. Since we cannot constrain this parameter at all, we conclude that it is an option to be further investigated. Another possibility may be that the pulsation changed direction, from retrograde (anti-rotation) to a prograde mode, changing $m$ from +1 to -1 . Since the observed frequency can be written as

$f_{\text {obs }}=f_{\text {puls }}-m * f_{\text {rot }}$,

it follows that the rotational period is $P_{\text {rot }}=15.4 \mathrm{~d}$ in this scenario. This possibility is appealing since, apart from the direction, the induced wind variability remains constant.

We cannot exclude the possibility that the pulsational mode did change, thereby changing the pulsational degree $l$ and $m$. Such changes could indeed cause period changes of the order a few percent. However, our identification of a specific pulsational mode $(l=1,|m|=1)$ loses its footing. We conclude that it is difficult to reconcile the long-term brightening with the period decrease. However, several suggestions are put forward. Either the stellar radius did decrease, and the increase of the massloss hid the accompanying temperature increase. Or, a mode change of $k$, or from $m=+1$ to $m=-1$, needs further investigation.

\subsection{Discussion and conclusion on the pulsational model}

Maeder (1985) and Maeder \& Schaller (1991) examined the theoretical status of pulsating WR stars. In He coreburning models the $\epsilon$ mechanism is expected to produce radial pulsation on a time scale of minutes to an hour. As to non-radial pulsations, Noels \& Scuflaire (1986) showed that these are expected due to the $\epsilon$ mechanism in the case of WR stars with hydrogen shell burning, but not for $\mathrm{H}$ - or He-burning cores, This may be applicable to WR 66 (Sect. 1; Rauw et al. 1996), but not for WR 46. The $\kappa$-mechanism, responsible for, e.g., $\beta$ - and $\delta$-Cepheid pulsation, may also induce radial instability of more massive $\left(>30 M_{\odot}\right)$ WR stars. Further research is necessary to determine whether pure He-stars, like WR 46, are also expected to show non-radial modes due to the $\kappa$-mechanism.

In addition, Glatzel et al.'s (1993) models of WR stars revealed the so-called strange-mode pulsation. Recent model calculations by Glatzel et al. (1999) showed that in the case of a low-mass WR star $\left(6\right.$ and $\left.14 M_{\odot}\right)$ the amplitude of the velocity field grows exponentially to a constant level of 100 to $200 \mathrm{~km} \mathrm{~s}^{-1}$, a small light amplitude of a few to 40 millimagnitudes, and a short pulsation period ( $100 \mathrm{~s}$ to $12 \mathrm{~min})$. We raise here the question whether the observed hourly periods may be related to predicted minute-like variability by way of a beating phenomenon. Note that change of a beat frequency requires a much smaller relative change of either of the constituting high frequencies.

It has been pointed out that a WR wind may smother the variability of the core. Matthews \& Beech (1987) argued that the effects of rapid pulsation $(P<12 \mathrm{hrs})$ should average out over the whole line-forming region, and should not appear as a net shift of the emission lines. In fact, we propose in Paper II that WR 46 is a borderline case, for which one cycle may be smoothed out, while another is not. Possibly, the observed short-term variability of WR 46 is common among WR stars, and happens to be observable in WR 46 because of its low-density wind. The study of other weak-lined WR stars will help answering this question. However, we point out that WR 46 is also singled out because of its long-term brightening, which probably would be detectable also with a denser wind. As a final remark, we note that pulsation is suggested to help the lift-off at the base of the wind; thus, a higher pulsational amplitude may enhance the mass-loss rate, in line with the observed changes.

In summary, the short-term photometric double-wave and radial velocity single-wave is indicative of a binary. Notwithstanding, it seems that it can be reconciled with non-radial pulsation as well. Note that the suggested pulsational mode $(l=1,|m|=1)$ has recently been identified in the S Dor-star (LBV) HD 160529 (Gäng 2000). The apparent disagreement between the long-term brightening and the period decrease may be removed by improved theoretical understanding of pulsations in WR stars. Therefore, we conclude that non-radial pulsations may be driving the photometric and spectroscopic shortterm variability of WR 46 .

\section{Conclusions}

We could not agree more with Kuhi (1968) when he claimed that "HD 104994 (WN3) is the strangest of all 
(WR stars)", albeit for completely different reasons. He meant the peculiar spectrum of WR 46: both the triangular shaped line profiles and the presence of O vi 3811-34. The triangular line-profiles are now understood as a consequence of a relatively low-density WR wind (CSH). And the peculiar oxygen line-complex is probably not due to peculiar oxygen abundance as advocated by CSH, but due to extreme susceptibility of this transition to low-density regions (Paper II).

To us, WR 46 is "strange" because of its short-periodic photometric double-wave, its peculiar radial-velocity behaviour, from being constant to large amplitude cycles, and its long-term light variations possibly correlated with period changes and variations of mass-loss rate. Based on our observations we proposed (Paper II) that the (dis)appearance of radial velocity cycles may be related to the observed large time-delays of the radial velocity.

Because of the complexity of the observed phenomena, we developed a phenomenological model for the shortterm double-wave variability, free from interpretation as much as possible. In this model the wind has an ellipsoidal density distribution, without specifying what induces the distortion. The geometrical distortion of the stellar wind rotates with the photometric double-wave period. We show that close to the star the distortion of the emission layers increases when going outwards, which explains the typical colour and line flux behaviour. Moreover, the outer layers trail behind.

The obvious source of the distortion is a close interacting binary. If so, we infer that the companion may be a non-compact low-mass object, either a secondary MS star $\left(\leq 0.7 M_{\odot}\right)$, a He-star $\left(\leq 4 M_{\odot}\right)$, or a WD. For the lowest mass companion, the accompanying WR mass also needs to be low $\left(4\right.$ or $5 M_{\odot}$ ). However, the large period change between 1989 and 1991 is problematic for any double-star model, assuming the photometric periods represent the orbital period. Only for small masses of the components and large WR radius the conservation laws are not violated. For that case, several mechanisms are proposed that might accomplish the transfer of energy and angular momentum on such a short time scale. Yet, if the period change is interpreted as orbital decay, it practically invalidates the double-star models.

Therefore, we propose that WR 46 may be a nonradial pulsator. The occurence of an additional period in the large data set of $1989\left(P_{\mathrm{x}}\right)$ supports this suggestion. Presumably, the pulsating surface affects the massloss and, thereby, the wind density. We suggest two pulsational modes which may cause a photometric double-wave with a radial-velocity single-wave, hereby mimicking the variability of a binary. The interpretation of the long-term brightening and accompanying period decrease is not selfevident, but several suggestions are put forward.

Therefore, the enigma rests therein, that the obvious interpretation of the observed short-term variability (a binary) is in conflict with the observed long-term variation of WR 46. To decide between pulsation, or binarity, or a combination of both, it is necessary to perform simulta- neous photometric and spectroscopic monitoring around the globe during more than a week. The relation between time-delay and stand-stills, suggested in Paper II, can then be investigated much better. If, in the long run, the light maxima do coincide with the extremes of the radial velocity, and the period continues to decrease, possibly with sudden jumps, the hypothesis of spiral-in would be strengthened. Then, WR 46 would allow the study of very rapid binary evolution in the coming years to decades. The pulsational model, however, predicts the occurence of more random periods. In that case WR 46 would be a prime candidate to investigate the relation of stellar pulsation and WR stellar wind, and maybe even the WR stellar interior by means of astero-seismology.

Although we may have lifted a corner of the veil, it seems that we have uncovered a deeper blanket of secrecy around WR 46.

Acknowledgements. We are grateful to Marten van Kerkwijk for extensive discussion and for pointing out the possible appearances of various pulsational modes. We are indebted to Paul Crowther and George Meynet for WR atmosphere and interior specifics, respectively. We thank Gertjan Savonije, Coen Schrijvers, Jeroen de Jong, Slavek Rucinski, and the referee, Tony Moffat, for their invaluable remarks.

\section{References}

Antokhin, I. I. 1996, in Wolf-Rayet Stars in the Framework of Stellar Evolution, Proc. 33rd Liège Int. Astroph. Coll., ed. J.-M. Vreux, et al. (Liège: Univ. of Liège), 177

Antokhin, I. I., Bertrand, J.-F., Lamontagne, R., et al. 1995, AJ, 109, 817

Bratschi, P., \& Blecha, A. 1996, A\&A, 313, 537

Brown, J. C., Richardson, L., Antokhin, I., et al. 1995, A\&A, 295,725

Cieslinski, D., Diaz, M. P., \& Steiner, J. A. 1999, AJ, 117, 534

Crowther, P. A., Smith, L. J., \& Hillier, J. D. 1995, A\&A, 302, $457(\mathrm{CSH})$

Drechsel, H., Rahe, J., Wargau, W., \& Wolf, B. 1982, A\&A, 110,246

Drechsel, H., Haas, S., Lorenz, R., \& Gayler, S. 1995, A\&A, 294,723

Drechsel, H., \& Lorenz, R. 1993, IBVS, 3868

Duijssens, M. F. J., van der Hucht, K. A., van Genderen, A. M., et al. 1996, A\&AS, 119, 37

Gäng, T. 2000, Thesis, University of Heidelberg

van Genderen, A. M., Verheijen, M. A. W., van Kampen, E., et al. 1991, in Wolf-Rayet Stars and Interrelations with Other Massive Stars in Galaxies, ed. K. A. van der Hucht, \& B. Hidayat (Kluwer, Dordrecht), IAU Symp., 143, 129

van Genderen, A. M., \& van der Hucht, K. A. 1986, A\&A, 162, 109

Georgiev, L., Koenigsberger, G., Ivanov, M., et al. 1999, A\&A, 347, 583

Glatzel, W., Kiriakidis, M., \& Fricke, K. J. 1993, MNRAS, 262, L7

Glatzel, W., Kiriakidis, M., Chernigovskij, S., \& Fricke, K. J. 1999, MNRAS, 303, 116

Hanson, M. M., Still, M. D., \& Fender, R. P. 2000, ApJ, 541, 308

Heger, A., Langer, N., \& Woosley, S. E. 2000, ApJ, 528, 368 
Heger, A., \& Langer, N. 2000, ApJ, 544, 1016

Herzceg, T. J. 1992, in Evolutionary Processes in Interacting Binary Stars, ed. Y. Kondo, R. F. Sistero, \& S. Polidan (Kluwer, Dordrecht), IAU Symp., 151, 310

Herczeg, T. J., \& Drechsel, H. 1985, ApSS, 114, 1

Huang, R. Q. 1999, A\&A, 344, 891

Jones, T. J., Gehrz, R. D., Kobulnicky, H. A., et al. 1994, AJ, 108,605

Kelley, R. L., Rappaport, S., Clark, G. W., \& Petro, L. D. 1983, ApJ, 268, 790

van Kerkwijk, M. H. 1993, Thesis, University of Amsterdam, 130

van Kerkwijk, M. H., Charles, P. A., Geballe, T. R., et al. 1992, Nature, 355, 703

van Kerkwijk, M. H., Geballe, T. R., King, D. L., et al. 1996, A\&A, 314, 521

Kuhi, L. V. 1968, in Wolf-Rayet Stars, ed. K. B. Gebbie, \& R. N. Thomas (NBS Special Publ.), 307, 103

Lamers, H. J. G. L. M., \& Cassinelli, J. P. 1999, Introduction to Stellar Winds (Cambridge University Press, Cambridge, UK), 29

Langer, N. 1989, A\&A, 210, 93

Langer, N., \& Heger, A. 1999, in Wolf-Rayet Phenomena in Massive Stars and Starburst Galaxies, ed. K. A. van der Hucht, G. Koenigsberger, \& P. R. J. Eenens, Proc. IAU Symp., 193 (San Francisco: ASP), 187

Maeder, A. 1985, A\&A, 147, 300

Maeder, A., \& Schaller, G. 1991, in Wolf-Rayet Stars and Interrelations with Other Massive Stars in Galaxies, ed. K. A. van der Hucht, \& B. Hidayat (Kluwer, Dordrecht), IAU Symp., 143, 167

Marchenko, S. V., Moffat, A. F. J., van der Hucht, K. A., et al. 1998, A\&A, 331, 1022

Marchenko, S. V., Arias, J., Barbà, R., et al. 2000, AJ, 120, 2101, (MAB)

Mason, K. O., Becklin, E. E., Blankenship, L., et al. 1976, ApJ, 207, 78

Massey, P., \& Conti, P. S. 1981, ApJ, 244, 173

Matthews, J. M., \& Beech, M. 1987, ApJ, 313, L25

Meynet, G. 1998, in Properties of Hot, Luminous Stars, Proc. Boulder-Münich Workshop II, ed. I. D. Howarth (ASP: San Fransisco), ASP Conf. Ser., 131, 96

Meynet, G., \& Maeder, A. 2000, A\&A, 361, 101

Mitra, A. 1998, ApJ, 499, 385

Moffat, A. F. J. 1982, in Wolf-Rayet Stars: Observations, Physics, Evolution, ed. C. de Loore, \& A. J. Willis (Dordrecht: Reidel), Proc. IAU Symp., 99, 263
Moffat, A. F. J., \& Marchenko, S. V. 1996, A\&A, 305, L29

Morel, T., St-Louis, N., \& Marchenko, S. V. 1997, ApJ, 482, 470

Morel, T., St-Louis, N., Moffat, A. F. J., et al. 1998, ApJ, 498, 413

Morel, T., Marchenko, S. V., Eenens, P. R. J., et al. 1999, ApJ, 518,428

Niemela, V. S., Barbá, R. H., \& Shara, M. M. 1995, in WolfRayet stars: Binaries, Colliding Winds, Evolution, ed. K. A. van der Hucht, \& P. M. Williams (Kluwer, Dordrecht), IAU Symp., 163, 245

Noels, A., \& Scuflaire, R. 1986, A\&A, 161, 125

Patterson, J., Kemp, J., Saad, J., et al. 1997, PASP, 109, 468

Pollock, A. M. T. 1995, in Wolf-Rayet Stars: Binaries, Colliding Winds, Evolution, ed. K. A. van der Hucht, \& P. M. Williams (Kluwer, Dordrecht), Proc. IAU Symp., 163, 429

Portegies Zwart, S., \& Verbunt, F. 1996, A\&A, 309, 179

Rauw, G., Gosset, E., Manfroid, J., Vreux, J.-M., \& Claeskens, J.-F. 1996, A\&A, 306, 783

Schaerer, D., \& Maeder, A. 1992, A\&A, 263, 129

Schmutz, W. 1997, A\&A, 321, 268

Schmutz, W., Geballe, T. R., \& Schild, H. 1996, A\&A, 311, L25

Šimon, V., \& Mattei, J. A. 1999, A\&AS, 139, 75

Steiner, J. E., Cieslinski, D., Jablonski, F. J., \& Williams, R. E. 1999 A\&A, 351, 1021

Stella, L., White, N. E., \& Rosner, R. 1986, ApJ, 308, 669

Sterken, C., \& Breysacher, J. 1997, A\&A, 328, 269

Stevens, I. R., \& Willis, A. J. 1988, MNRAS, 234, 783

St-Louis, N., Dalton, M. J., Marchenko, S. V., et al. 1995, ApJ, 452, L57

Tovmassian, H. M., Navarro, S. G., \& Cardona, O. 1996, AJ, 111,306

Vanbeveren, D., Van Rensbergen, W., \& de Loore, C. 1998, The Brightest Binaries, Astrophysics \& Space Science Library 232 (Kluwer Academic Publishers, Dordrecht)

Veen, P. M., \& Wieringa, M. H. 2000, A\&A, 363, 1026

Veen, P. M., Van Genderen, A. M., van der Hucht, K. A., et al. 2002a, A\&A, 385, 585 (Paper I)

Veen, P. M., Van Genderen, A. M., Crowther, P. A., \& van der Hucht, K. A. 2002b, A\&A, 385, 600 (Paper II)

Wagner, R. M., Kreidl, T. J., Bus, S. J., \& Williams, W. 1989, ApJ, 346, 971

Wilson, O. C. 1939, PASP, 51, 55

Witte, M. G., \& Savonije, G. J. 1999, A\&A, 350, 129 